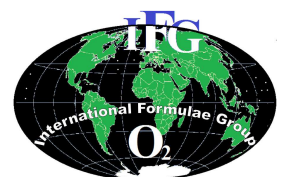

Available online at http://ajol.info/index.php/ijbcs

Int. J. Biol. Chem. Sci. 8(4): 1730-1741, August 2014

ISSN 1997-342X (Online), ISSN 1991-8631 (Print)
International Joumal

of Biological and

Chemical Sciences

Original Paper

http://indexmedicus.afro.who.int

\title{
Étude de quelques Trachelomonas Ehr. (Euglenophyta) nouveaux ou peu connus pour la Côte d'Ivoire
}

\author{
Kouhété Philippe DA ${ }^{1 *}$, Soulemane BAMBARA ${ }^{2}$ et Koffi N'GUESSAN ${ }^{1}$ \\ ${ }^{1}$ Laboratoire de Botanique, UFR Biosciences, Université Félix Houphouët-Boigny, 22 BP 582 Abidjan 22, \\ Côte d'Ivoire. \\ ${ }^{2}$ Projet PNUD/FEM IVC/94/G 31 «Lutte Contre les Végétaux Aquatiques Envahissant les Plans d'Eau pour \\ Améliorer/Restaurer la Diversité Biologique » CIAPOL 01 BP V 153 Abidjan 01, Côte d'Ivoire. \\ *Auteur correspondant ; E-mail : philippe_da@yahoo.fr; Tél : +2250797 5892.
}

\section{RÉSUMÉ}

La présente étude taxinomique, effectuée au microscope photonique, porte sur des prélèvements effectués au filet à plancton de $20 \mu \mathrm{m}$ de vide de maille dans les rivières Agnéby et Bia, dans le Sud de la Côte d'Ivoire. Elle fait suite aux cinquante-huit taxons du même genre Trachelomonas Ehr. (Euglenophyta) déjà décrits au microscope électronique à balayage et vise à faire connaître 22 taxons dont 19 non encore signalés et 3 peu connus (rares) en Côte d'Ivoire: Trachelomonas armata (Ehrenberg) Stein fo. inevoluta Deflandre, $T r$ dubia Swirenko emend. Deflandre et Tr lefevrei Deflandre. Les échantillons ont été prélevés entre 1995 et 2000. Sur les vingt-deux (22) taxons décrits, on compte onze (11) espèces, sept (7) variétés et quatre (4) formes.

(c) 2014 International Formulae Group. All rights reserved.

Mots clés : Taxinomie, Trachelomonas, Euglenophyta, eau douce, Sud Côte d'Ivoire.

\section{INTRODUCTION}

Le genre Trachelomonas Ehrenberg réunit des algues unicellulaires, libres, mobiles grâce à un flagelle antérieur visible. Les cellules sont incluses dans une logette à paroi plus ou moins épaisse, fragile, de formes très variées. La surface de cette paroi est très diversement ornée et les détails de cette ornementation sont souvent difficiles à percevoir en microscopie photonique.

De très nombreuses publications ont déjà été produites de par le monde, en microscopie photonique comme électronique à balayage sur les Euglénophytes en général, et sur le genre Trachelomonas en particulier.
Dans la sous-région ouest-africaine, on peut citer entre autres travaux ceux de Bourrelly (1975) en Guinée Conakry, Couté \& Rousselin (1975) au Mali, Gerrath et Denny (1979) en Sierra Leone, Biswas et Nweze (1990) au Nigeria, Compère (1991) et Ba (2006) au Sénégal, Zongo (2007) au Burkina Faso, Djima (2013) au Niger. En Côte d'Ivoire, Bourrelly (1961) a décrit des algues dont des espèces du genre Trachelomonas. Après lui, Uherkovich et Rai (1977), Couté et Iltis 1981), Da (1992), Adou (1999), Ouattara et al. (2000), Da et al. (2009), NiamienÉbrottié (2010), Seu-Anoï (2012), Adon (2013), et Kouassi (2013) ont également 
publié sur ces algues; Couté et Iltis (1981), tout comme Da et al. (2009) ayant étudié les taxons de ce seul genre. La présente étude vise à améliorer la connaissance de ce genre et partant, de la biodiversité de ce pays.

\section{MATÉRIEL ET MÉTHODES}

Généralités sur la zone d'étude

Stations des échantillonnages

Les récoltes ont été effectuées à : Agboville-pont, Adahou-village, Armébé et Dabou-pont, sur la rivière Agnéby, Akressi, Diévisso-pont, Ayamé 1 et Aboisso, sur la rivière $\mathrm{Bia}$.

Ces stations sont toutes situées dans le Sud de la Côte d'Ivoire. La Figure 1 de leur localisation indique la situation géographique de la zone d'étude en Afrique et en Côte d'Ivoire (A) et celle des stations de prélèvement dans la zone d'étude $(\mathbf{B})$.

\section{Dans le bassin versant de la rivière Agnéby}

La station d'Agboville-pont est située dans le cours supérieur de la rivière, par 556' $\mathrm{N}$ et $4^{\circ} 13^{\prime} \mathrm{W}$, mesures effectuées au GPS 12 XL 12 CHANNEL GARMIN. Pendant la grande saison sèche (décembre-mars), ce cours d'eau est réduit à de petites flaques observables de place en place suivant un parcours sinueux. Sa profondeur ne dépasse alors guère $0,5 \mathrm{~m}$, mais peut atteindre $2 \mathrm{~m}$ en saison pluvieuse (avril-juillet pour la grande saison).

Les macrophytes angiospermiens et ptéridophytiques qu'on peut y observer sont : Azolla africana Desv. (Azollaceae), Cyperus difformis L. (Cyperaceae), Dactyloctaenium aegyptium (L.) P. Beauv. (Poaceae), Eichhornia crassipes (Mart.) Solm-Laub. (Pontederiaceae), Echinochloa pyramidalis (Lam.) Hitch. et Chase (Poaceae), Fimbristylis littoralis Gaud. (Cyperaceae), Lemna aequinoctialis Hegelm. (Lemnaceae), Ludwigia erecta (L.) Hara (Onagraceae), Ludwigia stenoraphe (Brendan) Hara (Onagraceae), Nymphaea lotus L.
(Nymphaeaceae), Sphenoclea zeylanica Gaertn. (Sphenocleaceae).

La station d'Adahou-village est située à $5 \mathrm{~km}$ au Sud-Ouest de la ville d'Agboville, par $5^{\circ} 54^{\prime} \mathrm{N}, 4^{\circ} 13^{\prime} \mathrm{W}$. C'est une petite mare permanente envahie par des macrophytes, et qui ravitaille le village en eau.

Sa flore macrophytique comprend: Ageratum conyzoides L. (Asteraceae), Alchornea cordifolia (Schum. et Thonn.) Müll. Arg. (Euphorbiaceae), Bambusa vulgaris Schrad. (Poaceae), Canna indica L. (Cannaceae), Chromolaena odorata (L.) R. King et H. Rob. (Asteraceae), Cyclosorus striatus (Schum.) Ching (Thelypteridaceae), Mimosa pudica L. (Mimosaceae), Panicum laxum Sw. (Poaceae), Panicum maximum Jacq. (Poaceae), Sida corymbosa R.E. Fries (Malvaceae), Sorghum arundinaceum Stapf (Poaceae), Panicum laxum étant l'espèce la plus abondante, suivie de Sorghum arundinaceum.

La station d'Armébé est située sur le cours inférieur de l'Agnéby, avec comme coordonnées géographiques $5^{\circ} 20^{\prime} \mathrm{N}$ et $4^{\circ} 19^{\prime} \mathrm{W}$. Elle est en eau permanente dont la profondeur moyenne est de $7 \mathrm{~m}$ environ. Comme macrophytes, on a: Bambusa vulgaris Schrad. (Poaceae), Ceratophyllum demersum L. (Ceratophyllaceae), Cyperus distans L. f. (Cyperaceae), Cyrtosperma senegalense (Schott) Engl. (Araceae), Eichhornia crassipes (Mart.) Solm-Laub. (Pontederiaceae), Ludwigia stolonifera (Guill. et Perr.) R.H. Raven (Onagraceae), Pistia stratiotes L. (Araceae), Salvinia nymphellula Desv. (Salviniaceae).

La station de Dabou-pont située à moins de $10 \mathrm{~km}$ d'Armébé, elle donne sur la lagune Ébrié. Ses coordonnées géographiques sont $5^{\circ} 18^{\prime} \mathrm{N}$ et $4^{\circ} 01^{\prime} \mathrm{W}$. Les macrophytes rencontrés sont: Acroceras zizanioides (Kunth) Dandy (Poaceae), Ceratophyllum demersum L. (Ceratophyllaceae), Cyclosorus striatus (Schum.) Ching (Thelypteridaceae), 
Echinochloa pyramidalis (Lam.) Hitch. et Chase (Poaceae), Ipomoea aquatica Forsk. (Convolvulaceae), Ipomoea cairica (L.) Sw. (Convolvulaceae), Lemna aequinoctialis Welw. (Lemnaceae), Ludwigia abyssinica A. Rich. (Onagraceae), Ludwigia stenoraphe (Brendan) Hara subsp. stenoraphe (Onagraceae), Pistia stratiotes L. (Araceae) et Salvinia nymphellula Desv. (Salviniaceae).

Dans le bassin versant de la rivière Bia

La station d'Akressi se trouve à $4 \mathrm{~km}$ après le village du même nom, sur le côté droit de la route menant à Bianouan. Ses coordonnées géographiques sont : $5^{\circ} 41^{\prime} \mathrm{N}$ et $3^{\circ} 05^{\prime} \mathrm{W}$.

Comme macrophytes, on y trouve: Alchornea cordifolia (Schum. et Thonn.) Müll. Arg. (Euphorbiaceae), Cecropia peltata L. (Cecropiaceae), Mimosa pudica L. (Mimosaceae), Panicum laxum Sw. (Poaceae), Pueraria phaseoloides (R.) B. (Fabaceae), Spermacoce verticillata L. (Rubiaceae), Panicum laxum étant de loin l'espèce la mieux représentée.

La station de Diévisso-pont : le pont sur l'axe Ayamé-Akressi enjambe un bras du lac d'Ayamé 1, en pleine zone forestière. Sa situation géographique est : $5^{\circ} 37^{\prime} \mathrm{N}$ et $3^{\circ} 07 \mathrm{~W}$. Les macrophytes qu'on y trouve sont : Alchornea cordifolia (Schum. et Thonn.) Müll. Arg. (Euphorbiaceae), Echinochloa pyramidalis (Lam.) Hitch. et Chase (Poaceae), Elaeis guineensis Jacq. (Arecaceae), Mitragyna ledermannii (K. Krause) Ridsdale (Rubiaceae), Nephrolepis biserrata (Sw.) Chott (Davalliaceae), Pistia stratiotes L. (Araceae), Pityrogramma calomelanos (L.) Link (Adiantaceae), Selaginella myosurus (Sw.) Alston (Selaginellaceae).

Le lac d'Ayamé 1 : les coordonnées géographiques de la station de prélèvement sont $5^{\circ} 36^{\prime} \mathrm{N}$ et $3^{\circ} 10^{\prime} \mathrm{W}$. La surface de l'eau est hérissée de troncs d'arbres morts. Les macrophytes rencontrés sont: Cassia siamea Lam. (Caesalpiniaceae), Echinochloa pyramidalis (Lam.) Hitch. et Chase (Poaceae), Mitragyna ledermannii (K. Krause) Ridsdale, Pistia stratiotes L. (Araceae).

La station de Bakro : cette station est à $5^{\circ} 33^{\prime} \mathrm{N}$ et $3^{\circ} 15^{\prime} \mathrm{W}$, dans un village de pêcheurs, en bordure du lac d'Ayamé. La surface de l'eau est également hérissée de troncs d'arbres morts. Parmi les macrophytes rencontrés, on peut citer: Ageratum conyzoides L. (Asteraceae), Alchornea cordifolia (Schum. et Thonn.) Mül. Arg. (Euphorbiaceae), Alternanthera pungens Kunth (Amaranthaceae), Bambusa vulgaris Schrad. (Poaceae), Bauhinia purpurea L. (Caesalpiniaceae), Cynodon dactylon (L.) Pers. (Poaceae), Cyperus compressus L. (Cyperaceae), Eleusine indica (L.) Gaertn. (Poaceae), Ipomoea quamoclit L. (Convolvulaceae), Justicia flava (Forssk.) Vahl (Acanthaceae), Melochia corchorifolia L. (Sterculiaceae), Panicum brevifolium L. (Poaceae), Paullinia pinnata L. (Sapindaceae), Rauvolfia vomitoria Afzel (Apocynaceae), Urena lobata L. (Malvaceae). La station d'Aboisso : avec $5^{\circ} 28^{\prime} \mathrm{N}$ et $\quad 3^{\circ} 12^{\prime} \mathrm{W}$ comme coordonnées géographiques, la station d'Aboisso est située dans le cours inférieur de la Bia et l'eau y coule sur un fond rocheux. Comme macrophytes rencontrés, on peut citer: Ageratum conyzoides L. (Asteraceae), Alchornea cordifolia (Schum. et Thonn.) Müll. Arg. (Euphorbiaceae), Anthocleista nobilis G. Don (Loganiaceae), Bombax buonopozense P. Beauv. (Bombacaceae), Chromolaena odorata (L.) R.M. King et H. Rob. (Asteraceae), Costus afer Ker Gawl. (Zingiberaceae), Cyclosorus dentatus (Forsk.) Ching (Thelypteridaceae), Elaeis guineensis Jacq. (Arecaceae), Heterotis rotundifolia (Sm.) J.-Fél. (Melastomataceae), Ipomoea mauritiana Jacq. (Convolvulaceae), Nephrolepis biserrata (Sw.) Schott (Davalliaceae), Piptadeniastrum africanum (Hook. f.) Brenan (Mimosaceae). 
Deux séries d'opérations ont été menées : des actions in situ, sur le terrain, et des travaux au laboratoire.

\section{Prélèvements et mesures in situ}

Des récoltes mensuelles destinées à l'étude qualitative des peuplements phytoplanctoniques ont été effectuées à l'aide d'un filet à plancton de $20 \mu \mathrm{m}$ de vide de maille dans les stations de prélèvement, d'août 1995 à octobre 1997. Elles ont été complétées par deux prélèvements ponctuels, le 22 septembre 2000 pour la mare d'Akressi, et le 24 septembre 2000 pour la mare d'Adahouvillage. Des échantillons d'eau de surface ont été prélevés à partir d'un seau de 10 litres et les algues recueillies par filtration au filet à plancton (qui constitue un moyen de concentration des organismes).

La fixation du matériel biologique a été réalisée immédiatement, avec une solution aqueuse de formaldéhyde (dont l'acidité a été neutralisée au borate de soude pour une meilleure conservation des échantillons) à la concentration finale de 5 p.c.

Les paramètres physico-chimiques ont également été mesurés mensuellement, lors des prélèvements, avec les sondes multiparamétriques YSI $600 \mathrm{XL}$ et AMEL 345, la transparence au disque de Secchi.

Concernant la station d'Akressi, un conductimètre $\mathrm{HACH} \quad \mathrm{CO} 150 \quad(\mathrm{pH}$, conductivité, salinité), un pHmètre WTW pH $330(\mathrm{pH} / \mathrm{mV}$, température) et un oxymètre WTW Oxi 197 (taux d'oxygène dissous, température) ont été nécessaires, ainsi qu'un disque de Secchi.

Pour Adahou-village, un conductimètre WTW LF 340 (conductivité et température), un pHmètre WTW pH $330 \quad(\mathrm{pH} / \mathrm{mV}$, température) et un oxymètre WTW Oxi 330 (taux d'oxygène dissous et température) ont été utilisés pour les études et mesures in situ de ces paramètres.
Pour des raisons de disponibilité du matériel, il n'a malheureusement pas été possible de toujours utiliser les mêmes instruments de mesure aux différentes stations, ce qui aurait eu l'avantage d'éviter de possibles distorsions des résultats.

Concernant les sels nutritifs (nitrates, nitrites, ions ammonium, phosphates), des échantillons d'eau ont été prélevés dans des flacons en polyéthylène de 1 litre et conservés au frais dans une glacière sur le terrain.

\section{Observations et analyses au laboratoire Observations et identifications}

$\mathrm{Au}$ laboratoire, les organismes phytoplanctoniques, sédimentés dans les piluliers, ont été prélevés sur le fond de ceuxci à l'aide d'une pipette et examinés au microscope photonique de marque WILD M 20 équipé d'une chambre claire pour les dessins. Un micromètre objet a permis les mensurations des cellules. Les dessins, effectués au crayon à papier, ont été repris à l'encre de Chine sur du papier calque. Les traits d'échelle des Figures 2 à 23 représentent $10 \mu \mathrm{m}$. L'identification des taxons au niveau spécifique et infraspécifique a été réalisée à partir des publications et ouvrages des auteurs suivants : Deflandre (1926), Huber-Pestalozzi (1955), Bourrelly (1961, 1985), Compère (1975, 1989), Gerrath et Denny (1979), Yacubson (1980), Tell et Zalocar De Domitrovic (1985), Tell et Conforti (1986), Conforti (1993), Mpawenayo (1996), Conforti et Pérez (2000), Conforti et Ruiz (2001).

\section{Analyses chimiques}

Les teneurs en nitrates ont été dosées selon la méthode AFNOR NF T 90-012 avec un système d'analyse M.A.S. (Multi Analyser System) et un spectrophotomètre SHIMADZU UV (visible) 120-02. Celles de l'azote ammoniacal ont été mesurées selon la méthode AFNOR NF T 90-110, par dosage 
titrimétrique direct. Les concentrations en ions orthophosphates $\left(\mathrm{PO}_{4}{ }^{3-}\right)$ ont été suivies selon la méthode NF T 90-023 à l'aide d'un spectrophotomètre SHIMADZU UV (visible) 120-02.

Les abréviations utilisées dans le texte sont les suivantes: L. : longueur; L.c.sp. : longueur avec les épines; L.s.sp. : longueur sans les épines; 1. : largeur ; 1.c.sp. : largeur avec les épines; 1.s.sp.: largeur sans les épines; L.ép. : longueur des épines; diam. ép. : diamètre des épines; loc. cit. : loco citato $=$ au lieu cité (cité plus haut); p.c. : pour cent.

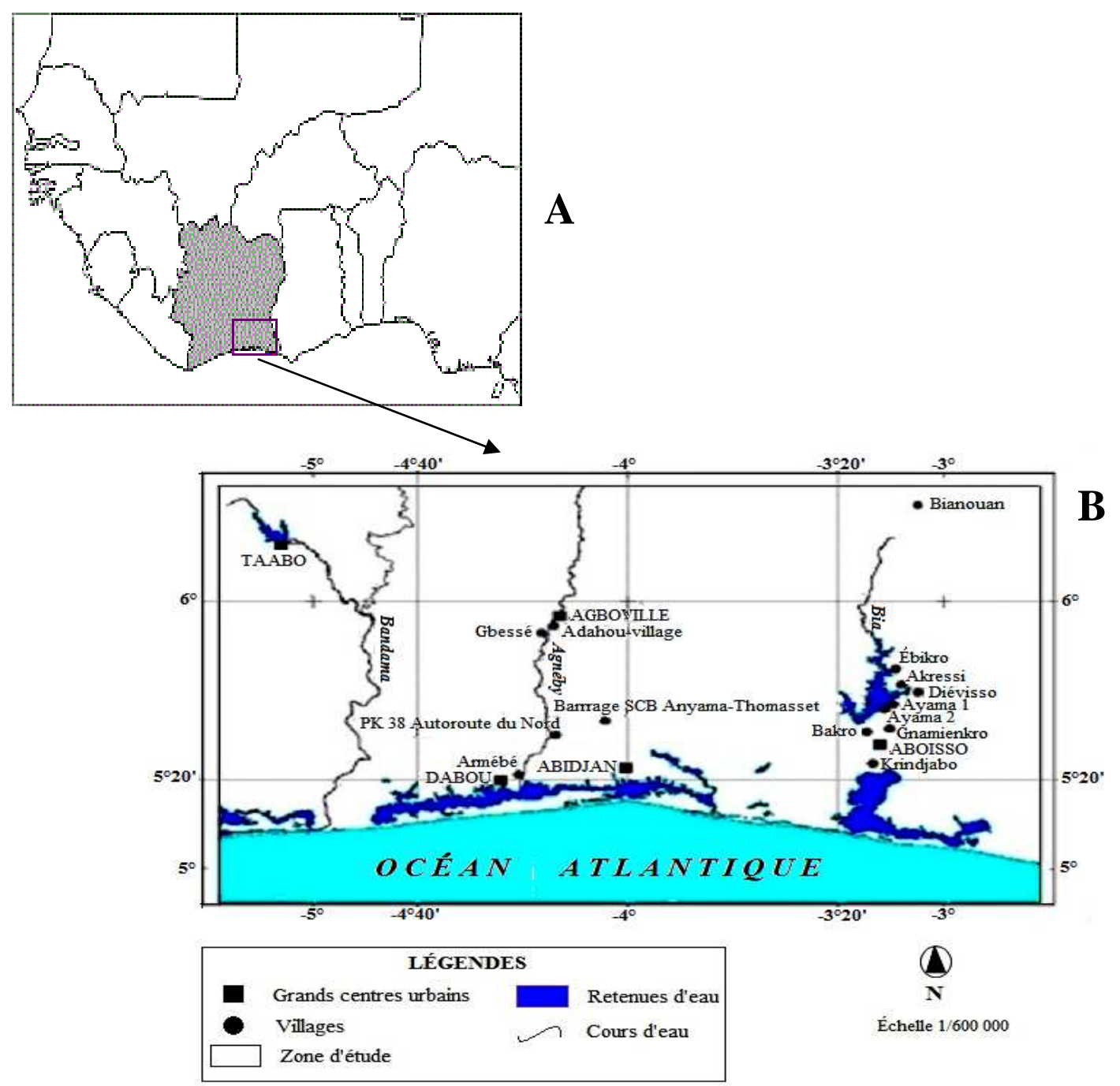

Figure 1 : Situation géographique de la zone d'étude en Afrique et en Côte d'Ivoire (A) et détails des stations de prélèvement (B). Modifié d'après BNETD-CCT 2006. 


\section{RÉSULTATS \\ Données écologiques}

À Agboville-pont, la température de l'eau était de $27,5{ }^{\circ} \mathrm{C}$ en moyenne, la moyenne de $\mathrm{pH}$ de 6,85 , celle du taux d'oxygène dissous de $2,7 \quad \mathrm{mg} \mathrm{L}^{-1}$, la conductivité de $174,8 \mu \mathrm{S} \mathrm{cm}{ }^{-1}$, la moyenne de salinité de 0,174 p.m. La transparence était de $0,50 \mathrm{~m}$ en moyenne. Pendant la même période, la teneur moyenne des nitrates était de $0,054 \mathrm{mg} \mathrm{L}^{-1}$, celles des nitrites de 0,181 $\mathrm{mg} \mathrm{L}^{-1}$, celle de l'azote ammoniacal de 0,276 $\mathrm{mg} \mathrm{L}^{-1}$ et celle des orthophosphates de 0,115 $\mathrm{mg} \mathrm{L}^{-1}$.

À Adahou-village, la température de l'eau a été de $26,3{ }^{\circ} \mathrm{C}$, le $\mathrm{pH}$ de 7,05 , le taux d'oxygène dissous de $7,9 \mathrm{mg} \mathrm{L}^{-1}$, la conductivité de $1,4 \mu \mathrm{S} \mathrm{cm}^{-1}$, la transparence non mesurable.

À Armébé, la température de l'eau était de $26,4{ }^{\circ} \mathrm{C}$ en moyenne, le $\mathrm{pH}$ moyen de 7,00 , la moyenne du taux d'oxygène dissous de $3,0 \mathrm{mg} \mathrm{L}^{-1}$, celle de la conductivité de $107,0 \mu \mathrm{S} \mathrm{cm}^{-1}$, celle de la transparence de 0,35 m.

À Dabou-pont, la température moyenne de l'eau a été de $26,5{ }^{\circ} \mathrm{C}$, le $\mathrm{pH}$ moyen de 7,04 , le taux d'oxygène dissous moyen de 2,9 $\mathrm{mg} \mathrm{L} \mathrm{L}^{-1}$, la moyenne de conductivité de 104,5 $\mu \mathrm{S} \mathrm{cm}^{-1}$, celle de la salinité de 0,240 p.m., celle de la transparence de $0,55 \mathrm{~m}$. Dans le même temps, la teneur moyenne en nitrates était de $0,28 \mathrm{mg} \mathrm{L}^{-1}$, celle des nitrites de 0,003 $\mathrm{mg} \mathrm{L}^{-1}$.

À Akressi, la température de l'eau était de $29,9{ }^{\circ} \mathrm{C}$, le $\mathrm{pH}$ de 8,32 , le taux d'oxygène dissous de 7,2 $\mathrm{mg} \mathrm{L}^{-1}$, la salinité nulle, la conductivité de $153,4 \mu \mathrm{S} \mathrm{cm}^{-1}$, la transparence de $0,15 \mathrm{~m}$.

À Diévisso-pont, aucun paramètre physico-chimique n'a pu être mesuré du fait de l'inaccessibilité de cette station.

À Ayamé 1, la température moyenne de l'eau était de $28,4{ }^{\circ} \mathrm{C}$, le $\mathrm{pH}$ moyen de 7,32 , la teneur moyenne en oxygène dissous de $6,5 \mathrm{mg}$ $\mathrm{L}^{-1}$, celle de la conductivité de $80,4 \mathrm{mg} \mathrm{L}^{-1}$, celle de la salinité nulle, la transparence moyenne de 1,06 $\mathrm{m}$. La teneur moyenne en nitrates a été de $0,30 \mathrm{mg} \mathrm{L}^{-1}$, celle des nitrites de $0,003 \mathrm{mg} \mathrm{L}^{-1}$, celle de l'azote ammoniacal de $0,50 \mathrm{mg} \mathrm{L}^{-1}$, et celle des orthophosphates de $1,19 \mathrm{mg} \mathrm{L}^{-1}$.

À Bakro, la température moyenne de l'eau a été de $29,9^{\circ} \mathrm{C}$, le $\mathrm{pH}$ moyen de 7,54 , le taux moyen d'oxygène dissous de $5,2 \mathrm{mg} \mathrm{L}^{-1}$, la conductivité moyenne de $59,0 \mu \mathrm{S} \mathrm{cm} \mathrm{cm}^{-1}$, la salinité nulle, la transparence moyenne de $1,01 \mathrm{~m}$. La teneur moyenne en nitrates a été de $0,30 \mathrm{mg} \mathrm{L}^{-1}$, celle des nitrates de $0,001 \mathrm{mg} \mathrm{L}^{-}$ 1 , celle de l'azote ammoniacal de $0,45 \mathrm{mg} \mathrm{L}^{-1}$ et celle des orthophosphates de $0,66 \mathrm{mg} \mathrm{L}^{-1}$.

À Aboisso, la température moyenne de l'eau était de $27,6^{\circ} \mathrm{C}$, le $\mathrm{pH}$ de 7,00 , la teneur moyenne en oxygène dissous de $9,1 \mathrm{mg} \mathrm{L}^{-1}$, la conductivité moyenne de $89,2 \mu \mathrm{S} \mathrm{cm} \mathrm{cm}^{-1}$, la salinité moyenne de 0,001 p.m., la transparence de $0,55 \mathrm{~m}$. La teneur moyenne en nitrates a été de $0,30 \mathrm{mg} \mathrm{L}^{-1}$, celle des nitrites de $0,001 \mathrm{mg} \mathrm{L}^{-1}$, celle de l'azote ammoniacal de $0,56 \mathrm{mg} \mathrm{L}^{-1}$ et celle des orthophosphates de $1,08 \mathrm{mg} \mathrm{L}^{-1}$.

\section{Description des algues observées Famille des Euglenaceae Genre Trachelomonas Ehrenberg}

Trachelomonas acanthophora Stokes var. speciosa (Deflandre) Balech

[= Trachelomonas speciosa Deflandre]

Logette largement fusiforme ; flancs largement arrondis convergeant rapidement à l'avant vers le col, à l'arrière vers la queue ; col court, droit, orné de 4-6 fortes épines pointues, divergentes ; queue creuse, conique, pointue, ou bien ouverte à son extrémité fourchue ou présentant 3-5 épines; paroi ornée d'épines coniques, peu nombreuses, assez distantes les unes des autres.

Dimensions : L.c.sp. : 50-51,7 $\mu \mathrm{m}$; 1.s.sp. : 18,8-20,5 $\mu \mathrm{m}$.

Distribution géographique: Venezuela, Argentine. Lieu de récolte : Diévisso-pont.

Trachelomonas anulifera (Fritsch et Rich) Huber-Pestalozzi

[= Trachelomonas rugulosa fa. Fritsch et Rich]. 
Logette sphérique à paroi ornée de stries horizontales régulièrement disposées. Dimensions : 18-20,8 $\mu \mathrm{m}$ de diamètre.

Distribution géographique: Afrique du Sud. Lieu de récolte : Diévisso-pont.

Trachelomonas armata (Ehrenberg) Stein fo. inevoluta Deflandre

Diffère du type et des autres variétés et formes par l'absence totale d'épines; logette largement ellipsoïdale, sans col ou à pore entouré d'un épaississement annulaire ; paroi lisse.

Dimensions : L. : $35 \mu \mathrm{m}$; 1. : 25-26,5 $\mu \mathrm{m}$.

Distribution géographique: probablement subcosmopolite. En Afrique: Burundi. Lieu de récolte : mare d'Adahou-village.

Trachelomonas armata var. longa Deflandre

Logette plus étroite que le type ; pôles parfois très légèrement aplatis ; pôle antérieur pourvu d'un pore sans col, orné de quelques courtes épines ; épines postérieures épaisses ; paroi finement ponctuée.

Dimensions : L.c.sp. : $43 \mu \mathrm{m}$; L.s.sp. : 36 $\mu \mathrm{m} ; 1$ : 26-28,5 $\mu \mathrm{m}$; L.ép. : 5-11 $\mu \mathrm{m}$; diam.ép. : 1-2,5 $\mu \mathrm{m}$.

Distribution géographique: Argentine, Belgique, Burundi, France. Lieu de récolte : mare d'Adahou-village.

Trachelomonas caudata (Ehrenberg) Stein

Logette ellipsoïdale assez étroite ; côtés peu arqués, s'atténuant peu à peu à l'avant et à l'arrière; pore large, coiffé d'un col tronconique; délimitation entre col et corps cellulaire souvent difficile à faire, mais visible cependant; partie postérieure munie d'une queue creuse, droite ou courbée, paraissant continuer les flancs de la logette, ou nettement distincte; paroi couverte d'épines pointues, courtes. Cette forme rappelle la Figure 648 de Deflandre (1926).

Dimensions : L. : 44,1 $\mu \mathrm{m}$; 1. : 19,1 $\mu \mathrm{m}$.

Distribution géographique : cosmopolite. Ouattara et al. (2000) ont décrit la même espèce, mais avec une forme qui diffère de la nôtre, raison de la présente description. Lieu de récolte: Agboville-pont sur la rivière Agnéby.
Trachelomonas cervicula Stokes

[= T. volvocina Ehrenberg var. cervicula (Stokes) Lemmermann].

Espèce à logette sphérique ou subsphérique, lisse ou ponctuée, de différentes couleurs, à pore pourvu d'un tube intérieur cylindrique, de longueur (4 à $8 \mu \mathrm{m}$ ) et d'inclinaison variables.

Dimensions : L. : $31 \mu \mathrm{m}$; 1. : $29,8 \mu \mathrm{m}$; tube long de 6-7 $\mu \mathrm{m}$.

Distribution géographique : cosmopolite. Lieu de récolte : Aboisso, mare d'Akressi.

Trachelomonas conica Playfair fo. conica

Logette cylindroconique; partie antérieure subrectangulaire, côtés parallèles, angles largement arrondis; partie postérieure conique, côtés légèrement arrondis, pôle postérieur obtus arrondi; pore sans col ou avec un col court ; paroi lisse.

Dimensions : L. : 27,9 $\mu \mathrm{m}$; 1. : 13,2 $\mu \mathrm{m}$.

Distribution géographique: Argentine, Australie, Venezuela. Lieu de récolte : Daboupont.

Trachelomonas conica var. conica fo. punctata Deflandre

Logette comme dans le type; paroi finement ponctuée.

Dimensions : L. : 13-27 $\mu \mathrm{m}$; 1. : 10,5-11 $\mu \mathrm{m}$. Distribution géographique: Mozambique, Venezuela. Lieu de récolte : mare d'Adahouvillage.

Trachelomonas cylindrica Ehrenberg sec. Playfair

Logette cylindrique, côtés parallèles, pôle postérieur largement arrondi, pôle antérieur un peu aplati ; pore pourvu d'un col bas ; paroi lisse.

Dimensions : L. : $18 \mu \mathrm{m}$; 1. : 10,5 $\mu \mathrm{m}$.

Distribution géographique : cosmopolite. Lieu de récolte : mare d'Akressi.

Trachelomonas dubia Swirenko emend. Deflandre var. dubia

[= T. dubia Swirenko 1914, Die Euglenaceengattung Trachelomonas. Arch. Hydrobiol. Planktonk. 9 (4) : 638, pl.19, fig. 20].

Logette ellipsoïdale-cylindrique ; flancs parallèles sur environ $1 / 3$ de leur 
longueur, parfois un peu arqués convergeant un peu vers la partie antérieure ; pôle antérieur toujours largement arrondi, un peu aplati ; pôle postérieur plus ou moins arrondi, parfois subconique ou même nettement acuminé (fo. acuminata) ; pore avec ou sans épaississement annulaire, toujours pourvu d'un col droit, cylindrique, haut, à section plane ; épaississement annulaire du pore souvent très développé et rétrécissant l'ouverture du pore qui est ainsi inférieure au diamètre du col ; paroi lisse.

Dimensions : L. : 21,5 $\mu \mathrm{m}$; 1. : $11 \mu \mathrm{m}$; L.col : $1 \mu \mathrm{m}$; diam. : $3 \mu \mathrm{m}$.

Distribution géographique : subcosmopolite. En Afrique : Algérie, Mozambique. Lieu de récolte : mare d'Akressi.

Trachelomonas dubia var. lata Deflandre [= T. euchlora (Ehrenberg.) Lemmermann in Skuja 1926].

Logette différant du type par ses dimensions plus grandes.

Dimensions : L. : $35 \mu \mathrm{m}$; 1. : 18,5 $\mu \mathrm{m}$; col

long de $4 \mu \mathrm{m}$, large de $6 \mu \mathrm{m}$.

Distribution géographique: subcosmopolite. En Afrique: Mozambique. Lieu de récolte: mare d'Adahou-village.

Trachelomonas elegans Conrad

Logette subsphérique, brunâtre, assez mince, simplement perforée à l'avant et hérissée de fines épines rayonnantes, courtes, serrées.

Dimensions : L. : $15 \mu \mathrm{m} ; 1$. : 13,5 $\mu \mathrm{m}$.

Distribution géographique: Belgique, Mozambique. Lieu de récolte: mare d'Akressi.

Trachelomonas hispida (Perty) Stein emend. Deflandre var. punctata Lemmermann

Logette ellipsoïdale comme dans le type, mais dépourvue d'épines; paroi finement et densément ponctuée.

Dimensions : L. : 23,5 $\mu \mathrm{m}$; 1. : 19,5 $\mu \mathrm{m}$.

Distribution géographique: Afrique du Sud, Allemagne, France, Lettonie, Ukraine, Lieu de récolte : mare d'Adahou-village.

Trachelomonas lefevrei Deflandre

Logette largement ellipsoïdale ou un peu ovoïde; côtés un peu arqués, pôles largement arrondis; pore toujours pourvu d'un col peu élevé, subcylindrique, à bord irrégulièrement et finement crénelé ; paroi peu densément ponctuée.

Dimensions : L. : 28,2-34,7 $\mu \mathrm{m}$; 1. : 19,7-24,4 $\mu \mathrm{m}$; col long de 1,7-3 $\mu \mathrm{m}$, large de 3,5-5,5 $\mu \mathrm{m}$.

Distribution géographique: Allemagne, Argentine, Belgique, Danemark, France, Suisse. Lieu de récolte : Armébé.

Trachelomonas niklewskii Drezepolski fo.

Logette ellipsoïdale allongée, couverte d'épines, mais différant du type par ses épines très peu nombreuses, disposées sans ordre, et par ses dimensions plus grandes. Dimensions : L. : $28,5 \mu \mathrm{m}$; 1. : 16,5 $\mu \mathrm{m}$ sans les épines (15-23 x 7,5-11 $\mu \mathrm{m}$ pour le type).

Distribution géographique: Pologne. Lieu de récolte : mare d'Akressi.

Trachelomonas pulcherrima Playfair var. pulcherrima

Logette ellipsoïdale très allongée, pôles largement arrondis, côtés différemment arqués; pore sans col ou avec un col bas; paroi lisse.

Dimensions : L. : 21,5 $\mu \mathrm{m}$; 1. : 13,5 $\mu \mathrm{m}$. Col haut de $2 \mu \mathrm{m}$, large de $3,5 \mu \mathrm{m}$.

Distribution géographique: subcosmopolite.

Lieu de récolte : mare d'Adahou-village.

Trachelomonas pulcherrima var. minor Playfair

Logette comme le type, mais plus petite.

Dimensions : L. : 15,5 $\mu \mathrm{m}$; 1. : 7,3-7,5 $\mu \mathrm{m}$.

Distribution géographique: subcosmopolite.

Lieu de récolte : mare d'Akressi.

Trachelomonas rugulosa Stein emend. Deflandre fo. rugulosa

[= T. stokesiana Palmer].

Logette sphérique, subsphérique ou largement ellipsoïdale, parfois un peu ovoïde, munie de stries épaisses, proéminentes, s'anastomosant par places, longitudinales, obliques ou spiralées, aboutissant en spirales autour du pore; pore à bord épaissi, quelquefois avec un col très bas; paroi d'épaisseur variable.

Dimensions : L. : 14,5-15 $\mu \mathrm{m}$; 1. : 13-14 $\mu \mathrm{m}$. Distribution géographique: cosmopolite. En Afrique: Mozambique, République Démocratique du Congo. Lieu de récolte: barrage d'Ayamé 1.

Trachelomonas rugulosa fo. parallela Tell et Zalocar 
Forme différant du type de l'espèce par les stries parallèles à l'axe longitudinal, avec quelques anastomoses.

Dimensions : L. : 15-19,7 $\mu \mathrm{m}$; 1. : 14,1-19,7 $\mu \mathrm{m}$.

Distribution géographique : pantropicale. Lieu de récolte : Aboisso, lac d'Ayamé 1, Bakro.

Trachelomonas scabra Playfair var. coberensis Deflandre

Logette subsphérique, parfois légèrement atténuée à la partie antérieure, munie d'un col cylindrique; paroi scabre.

Dimensions : L. : $16 \mu \mathrm{m}$; 1. : $14 \mu \mathrm{m}$. Col long de $2 \mu \mathrm{m}$, large de 3-4 $\mu \mathrm{m}$.

Distribution géographique: Venezuela. Lieu de récolte : Bakro.

Trachelomonas volzii Lemmermann var. cylindracea Playfair fo.

Logette un peu cylindrique, côtés parallèles, pôle postérieur largement arrondi, pôle antérieur conique, à côtés convergeant vers le col; col droit, robuste, muni d'un épaississement annulaire basal; paroi lisse. Cette variété diffère du type par l'existence de deux sortes de ponctuations : des ponctuations larges, de $1 \mu \mathrm{m}$ de diamètre, très éparses, irrégulièrement disposées, et des ponctuations fines, un peu plus denses.

Dimensions : L. : $35 \mu \mathrm{m}$; 1. : 15,5 $\mu \mathrm{m}$; col long de $3-5 \mu \mathrm{m}$, large de $3 \mu \mathrm{m}$.

Distribution géographique : Australie. Lieu de récolte : Armébé.

Trachelomonas woycickii Koczwara

Logette sphérique, densément couverte d'épines courtes; pore avec ou sans épaississement annulaire, sans col. Dimensions : diamètre de 26,5-27,5 $\mu \mathrm{m}$. Distribution géographique: France. Lieu de récolte : mare d'Adahou-village.

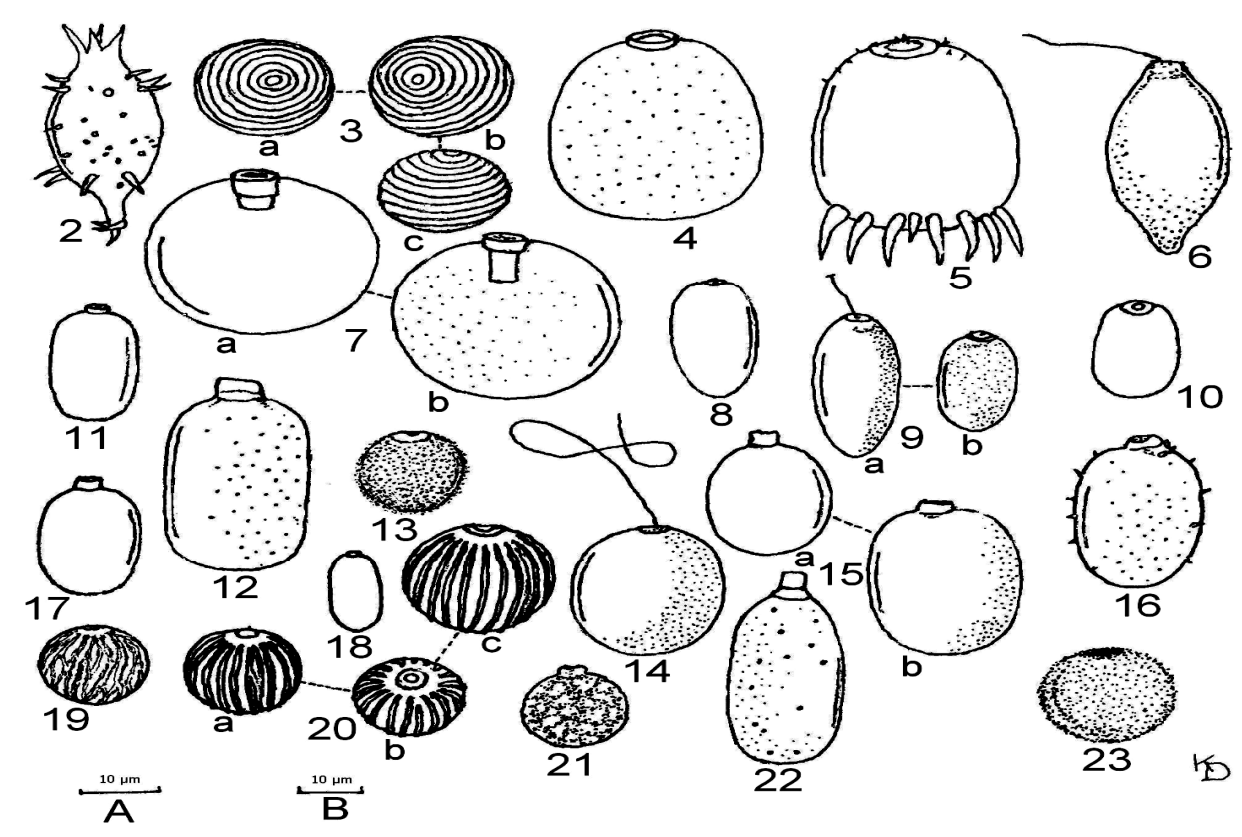

Figure 2 : Trachelomonas acanthophora Stokes var. speciosa (Defl.) Balech ; 3a, b, c : Tr. anulifera (Fritsch et Rich) Hub.-Pestal. ; Fig. 4 : Tr. armata (Ehr.) Stein fo. inevoluta Defl. ; Fig. 5 : Tr. armata var. longa Defl. ; Fig. $6:$ Tr. caudata (Ehr.) Stein ; Fig. 7a, b : Tr. cervicula Stokes ; Fig. $8:$ Tr. conica Playf. ; Fig. 9a, b : Tr. conica fo. punctata Defl. ; Fig. 10 : Tr. cylindrica Ehr. sec. Playf. ; Fig. 11 : Tr. dubia Swir. emend. Defl. ; Fig. 12 : Tr. dubia var. lata Defl. ; Fig. 13 : Tr. elegans Conrad ; Fig. 14 : Tr. hispida (Perty) Stein emend. Defl. var. punctata Lemm. ; Fig. 15a, b : Tr. lefevrei Defl. ; Fig. 16 : Tr. niklewskii Drez. fo. ; Fig. $17:$ Tr. pulcherrima Playf. ; Fig. $18: T r$. pulcherrima var. minor Playf. ; Fig. $19: T r$. rugulosa Stein emend. Defl. ; Fig. 20a, b, c : Tr. rugulosa fo. parallela Tell et Zalocar ; Fig. 21 : Tr. scabra Playf. var. coberensis Defl. ; Fig. 22 : Tr. volzii var. cylindracea Playf. Fig. $23: T r$. woycickii Kocz. (À l'exception des figures 2, 3c, 6, 8 et 15 qui sont à l'échelle $\mathrm{B}$, toutes les autres sont à l'échelle $\mathrm{A}$ ). 


\section{DISCUSSION}

La température de l'eau était généralement élevée et au-dessus de $25{ }^{\circ} \mathrm{C}$. Elle a varié entre 26,2 et $32,9{ }^{\circ} \mathrm{C}$, avec une moyenne de $27,8{ }^{\circ} \mathrm{C}$ et était favorable au développement des algues. Ces valeurs sont supérieures à celles notées par Kouassi (2013) à Adzopé $\left(23,3\right.$ à $\left.30,13{ }^{\circ} \mathrm{C}\right)$. Le $\mathrm{pH}$ a varié entre 6,85 et 8,32 (moyenne 7,26 ) et était pratiquement neutre à alcalin (exception faite d'Agboville-pont riche en matières organiques diverses en suspension, plutôt acide). La teneur moyenne en oxygène dissous était de $5,56 \mathrm{mg} \mathrm{L}^{-1}$, avec 2,9 et $9,1 \mathrm{mg} \mathrm{L}^{-1}$ comme valeurs extrêmes (sensiblement du même ordre de grandeur que celles des rivières Soumié, Éholié, Éhania et Noé (NiamienÉbrottié, 2010). L'eau était bien oxygénée. La conductivité, elle, a varié entre 1,4 et $174,8 \mu \mathrm{S}$ $\mathrm{cm}^{-1}$, avec une moyenne de $96,2 \mu \mathrm{S} \mathrm{cm}^{-1}$ inférieure à $100 \mu \mathrm{S} \mathrm{cm}^{-1}$ dénotant une eau très faiblement minéralisée, à l'exception de la station d'Agboville-pont où elle était faiblement minéralisée. Elle est plus faible que celle de la retenue d'eau d'Adzopé qui a varié entre 166,78 et $182,68 \mu \mathrm{S} \mathrm{cm} \mathrm{cm}^{-1}$ selon Adon (2013). La salinité, nulle dans la plupart des stations, était de 0,240 p.m. à Dabou-pont où l'influence de la lagune Ébrié proche se fait sentir. Sa valeur moyenne était de 0,051 p.m. La transparence, faible, est passée de $0,15 \mathrm{~m}$ à $1,06 \mathrm{~m}$ (moyenne 0,59). L'azote ammoniacal avait une concentration moyenne de $0,44 \mathrm{mg} \mathrm{L}^{-1}$, les extrêmes variant entre 0,228 à $0,56 \mathrm{mg} \mathrm{L}^{-1}$. À cette teneur moyenne supérieure à $0,3 \mathrm{mg} \mathrm{L}^{-1}$, cet ion intervient dans les phénomènes d'eutrophisation (Moreau, 1978). Les nitrates $\left(0,02-0,30 \mathrm{mg} \mathrm{L} \mathrm{L}^{-1}\right.$, moyenne $0,054 \mathrm{mg} \mathrm{L}^{-1}$ ) sont en plus faible concentration que ceux des rivières Soumié, Éholié et Noé (Niamien-Ébrottié (2010). Les nitrites $\left(0,001\right.$ à $0,181 \mathrm{mg} \mathrm{L}^{-1}$, avec une moyenne de $0,046 \mathrm{mg} \mathrm{L}^{-1}$ ) sont, à pareille concentration, une preuve de pollution organique. Les orthophosphates ont varié entre 0,115 et $1,19 \mathrm{mg} \mathrm{L}^{-1}$. Leur teneur moyenne $\left(0,761 \mathrm{mg} \mathrm{L}^{-1}\right)$, est élevée et stimule la croissance des algues.
Le genre Trachelomonas compte parmi les plus étudiés des Euglénophytes de ce pays. Ainsi, 147 taxons ont déjà été signalés en Côte d'Ivoire (Bourrelly, 1961 ; Uherkovich \& Rai, 1977; Couté \& Iltis, 1981 ; DA, 1992 ; Adou, 1999 ; Ouattara et al., 2000 ; Da et al., 2009 ; Niamien-Ébrottié, 2010 ; Seu-Anoï, 2012) ; Kouassi, 2013, dont 79 décrits en microscopie électronique à balayage par Couté \& Iltis (1981), Da et al. (2009).

\section{Conclusion}

Vingt-deux taxons comprenant onze espèces, sept variétés et quatre formes sont décrits dans la présente étude. À l'exception de 3 taxons: Trachelomonas armata fo. inevoluta, Tr. dubia et Tr. lefevrei déjà signalés mais peu connus (répandus), tous les 19 autres sont nouveaux pour la flore algologique de Côte d'Ivoire. Ils proviennent de récoltes effectuées entre 1995 et 2000 dans le Sud de la Côte d'Ivoire, à Agboville-pont, Adahou-village, Armébé et Dabou-pont, sur la rivière Agnéby, Akressi, Diévisso-pont, Ayamé 1 et Aboisso, sur la rivière Bia. Ils constituent une contribution à une meilleure connaissance des taxons spécifiques et infraspécifiques du genre Trachelomonas de ce pays.

Cette étude montre que malgré toutes les études déjà effectuées, ce pays n'a pas fini de nous surprendre par sa grande richesse algale qui, à n'en point douter, est loin d'avoir livré tous ses secrets.

\section{REFERENCES}

Adon MP. 2013. Variations spatiale et saisonnière du phytoplancton de la retenue d'eau d'Adzopé (Côte d'Ivoire) : composition, structure, biomasse et production primaire. Thèse de l'Université Nangui Abrogoua, Abidjan, p. 89.

Adou ARE. 1999. Contribution à la connaissance des algues de la lagune Tendo à N'Guiémé (Côte d'Ivoire). D.E.A. d'Écologie Tropicale (Option : 
Végétale), Université de Cocody, Abidjan, p. 95.

Ba N. 2006. La communauté phytoplanctonique du lac de Guiers (Sénégal): Types d'associations fonctionnelles et approches expérimentales des facteurs de régulation. Thèse de $3^{\mathrm{e}}$ cycle, Université Cheikh Anta Diop, Dakar, p. 144.

Biswas S, Nweze NO. 1990. Phytoplankton of Ogelube Opi, Anambra State, Nigeria. Hydrobiologia, 199 : 81-86.

Bourrelly P. 1961. Les Algues d'eau douce de la République de Côte d'Ivoire. Bull. I.F.A.N. sér. A, 23(2) : 283-374.

Bourrelly P. 1975. Quelques algues d'eau douce de Guinée. Bull. Mus. Nat. Hist. Nat., $3^{e}$ sér., Botanique, 20(276) : 1-71.

Bourrelly P. 1985. Les Algues d'Eau Douce. Initiation à la Systématique. Tome III : Les Algues Bleues et Rouges. Les Eugléniens, Peridiniens et Cryptomonadines. Société Nouvelle des Éditions Boubée : Paris.

Compère P. 1975. Algues de la région du lac Tchad III - Rhodophycées, Euglénophycées, Cryptophycées, Dinophycées, Chrysophycées, Xanthophycées. Cah. O.R.S.T.O.M., sér. Hydrobiol., 9(3) : 167-192.

Compère P. 1989. Flore Pratique des Algues d'Eau Douce de Belgique. 2: Pyrrhophytes, Raphidophytes, Euglénophytes. Jard. Bot. Nat. Belg.: Meise.

Compère P. 1991. Contribution à l'étude des algues du Sénégal. 1. Algues du lac de Guiers et du Bas-Sénégal. Bull. Jard. Bot. Nat. Belg., 61(3-4): 171-267.

Conforti VTD. 1993. Study of the Euglenophyta from Camaleão lake (Manaus-Brazil). I. Trachelomonas Ehr. Rev. Hydrobiol. Trop., 26(1): 3-18.

Conforti VTD, Pérez MC. 2000. Euglenophyceae of Negro River, Uruguay, South America. Archiv Hydrobiol. /Algological Studies, 97: 5978.
Conforti VTD, Ruiz L. 2001. Euglenophytes from Chunam Reservoir (South Korea). II. Trachelomonas Ehr. Arch. Hydrobiol. Suppl., 138/Algological Studies 102: 117145.

Couté A, Iltis A. 1981. Ultrastructure stéréoscopique de la logette de Trachelomonas (Algae, Euglenophyta) récoltés en Côte d'Ivoire. Rev. Hydrobiol. Trop., 14(2) : 115-133.

Couté A, Rousselin G. 1975. Contribution à l'étude des algues d'eau douce du Moyen Niger (Mali). Bull. Mus. Nat. Hist. Nat., $3^{e}$ sér., Botanique 21( 277): 73-175.

Couté A, Tell G. 2006. Some taxonomical and nomenclatural changes in the genus Trachelomonas Ehrenb. emend. Defl. (Euglenophyta). Archiv für Hydrobiol. Suppl. 165, Algol. Studies, 122: 73-82.

Da KP. 1992. Contribution à la connaissance $\mathrm{du}$ phytoplancton de la mare et du complexe piscicole du Banco (Côte d'Ivoire). Thèse de Doctorat de $3^{\mathrm{e}}$ cycle, Université Nationale de Côte d'Ivoire, Abidjan, p. 384.

Da KP, Mascarell G, Couté A. 2009. Étude au microscope électronique à balayage du genre Trachelomonas (Euglenophyta) dans le Sud-Est de la Côte d'Ivoire (Afrique de l'Ouest). Cryptogamie, Algol., 30(1) : 31-90.

Deflandre G. 1926. Monographie du genre Trachelomonas Ehr. A. Lesot : Nemours.

Djima IT. 2013. Les algues du fleuve Niger et les milieux humides connexes de l'Ouest du Niger. Thèse de Doctorat de l'Université Abdou Moumouni, Niamey, p. 202.

Gerrath JF, Denny P. 1979. Freshwater algae of Sierra Leone 1: Euglenophyta. Nova Hedwigia, 31(1-2): 525-565.

Huber-Pestalozzi G. 1955. Das Phytoplankton des Süßwassers. Systematik und Biologie. Euglenophyceen. In A. Thienemann, Die Binnengewässer, 16(4): 1-606.

Kim JT, Boo SM, Couté A. 2000. Taxonomic and Floristic Accounts of the Genus Trachelomonas Ehrenberg 1833 
(Euglenophyceae) from Korea. Korean J. Limnol., 33(2): 80-108.

Kouassi BAT. 2013. Taxinomie, composition floristique et dynamique spatiosaisonnière des algues périphytiques de la retenue d'eau d'Adzopé (Côte d'Ivoire). Thèse de l'Université Félix HouphouëtBoigny, Abidjan, p. 189.

Moreau D. 1978. Nitrification Dénitrification des eaux usées. Journées Informations Eaux, Poitiers, 28-29 septembre, T. 2; 1-10.

Mpawenayo B. 1996. Les eaux de la plaine de la Rusizi (Burundi): les milieux, la flore et la végétation algales. Acad. Roy. Sci. Outre-Mer. Classe Sci. nat. et médic. Mém. in- ${ }^{\circ}$, Nouv. Sér., 23(2): 1-236.

Niamien-Ébrottié JE. 2010. Composition et distribution spatiale et saisonnière des peuplements d'algues de quatre rivières du Sud-Est de la Côte d'Ivoire (Soumié, Éholié, Éhania et Noé). Thèse de l'Université d'Abobo-Adjamé, Abidjan, p. 118.

Ouattara A, Podoor N, Teugels GG, Gourène G. 2000. Les micro-algues de deux cours d'eau (Bia et Agnébi) de Côte d'Ivoire. Syst. Geogr. Pl., 70: 315-372.

Rino JA, Pereira MJ. 1988. Euglenophyta da Região Centro de Portugal I. Género Trachelomonas Ehrenb. 1833 emend.
Defl. 1926. Rev. Biol. U. Aveiro, 2: 129161.

Seu-Anoï NM. 2012. Structuration spatiale et saisonnière des peuplements phytoplanctoniques et variabilité des facteurs abiotiques dans trois complexes lagunaires de Côte d'Ivoire (Aby, Ébrié et Grand-Lahou). Thèse de Doctorat de l'Université Nangui Abrogoua, Abidjan, p. 135.

Tell G, Conforti VTD. 1986. Euglenophyta pigmentadas de la Argentina. Bibliotheca Phycologica, 75: 1-301.

Tell G, Zalocar De Domitrovic Y. 1985. Euglenophyta pigmentadas de la provincia del Chaco (Argentina). Nova Hedwigia, 41: 353-391.

Uherkovich G, Rai H. 1977. The phytoplankton of some waters in Ivory Coast (Africa). I. The Bouaké Reservoir. Arch. Hydrobiol., 81(2): 233-258.

Yacubson S. 1980. The Phytoplankton of Some Freshwater Bodies from Zulia State (Venezuela). Nova Hedwigia, 33: 279339.

Zongo F. 2007. Inventaire et systématique des micro-algues dulçaquicoles du réservoir de Bagré au Burkina Faso (Province du Boulgou). Thèse de Doctorat d'État ès Sciences Naturelles, Université de Ouagadougou, p. 214. 